\title{
Admissibility in De Morgan algebras
}

\author{
George Metcalfe $\cdot$ Christoph Röthlisberger
}

(C) Springer-Verlag 2012

\begin{abstract}
Characterizations of admissible quasi-identities, which may be understood as quasi-identities holding in free algebras on countably infinitely many generators, are provided for classes of De Morgan algebras and lattices.
\end{abstract}

Keywords De Morgan algebras · Structural completeness · Admissibility

\section{Introduction}

De Morgan algebras are algebraic structures $\langle A, \wedge, \vee, \neg$, $\perp, \top\rangle$ such that $\langle A, \wedge, \vee, \perp, \top\rangle$ is a bounded distributive lattice with bottom element $\perp$ and top element $T$, and $\neg$ is an involution (i.e., satisfies $\neg \neg x \approx x$ ) respecting the De Morgan laws $\neg(x \wedge y) \approx \neg x \vee \neg y$ and $\neg(x \vee y) \approx \neg x \wedge \neg y$. The class DMA of De Morgan algebras forms a variety containing just two proper non-trivial subvarieties: the class KA of Kleene algebras satisfying $x \wedge \neg x \leq y \vee \neg y$ and the class BA of Boolean algebras satisfying $x \leq y \vee \neg y$. The classes DML, KL, and BL of De Morgan, Kleene, and Boolean lattices are defined analogously by omitting the constants $\perp$ and $T$ from the language.

De Morgan lattices were first studied by Moisil (1935) and Kalman (1958), and subsequently, with or without the constants $\perp$ and $T$, by many other researchers. In particular, the quasivariety lattice of De Morgan lattices has been

G. Metcalfe $(\bowtie) \cdot$ C. Röthlisberger

Mathematics Institute, University of Bern,

Sidlerstrasse 5, 3012 Bern, Switzerland

e-mail: george.metcalfe@math.unibe.ch

C. Röthlisberger

e-mail: roethlisberger@math.unibe.ch fully characterized by Pynko (1999) (there are just seven non-trivial quasivarieties), while the more complicated (infinite) quasivariety lattice of De Morgan algebras has been investigated by Gaitán and Perea (2004). As is well known, DMA is generated by the diamond algebra $\mathbf{D}_{\mathbf{4}}, \mathrm{KA}$ by the three-valued chain $\mathbf{C}_{\mathbf{3}}$, and $\mathrm{BA}$ by the two-valued algebra $\mathbf{C}_{2}$. However, $\mathrm{KA}$ is also generated by the standard fuzzy algebra $\langle[0,1], \min , \max , 1-x, 0,1\rangle$, and DMA is generated by the fuzzy interval algebra $\langle\{(a, b) \mid a, b \in$ $[0,1] ; a \leq b\}, \wedge, \vee, \neg,(0,0),(1,1)\rangle$ where $\wedge$ and $\vee$ are min and max calculated component-wise and $\neg(a, b)=(1-$ $b, 1-a)$. These classes have therefore received considerable attention in the fuzzy logic literature (see, e.g., Gehrke et al. 2003). We note, moreover, that De Morgan algebras provide an underlying involutive lattice structure for the algebras of substructural logics such as R-mingle, Łukasiewicz logic, and multiplicative additive linear logic (see, e.g., Galatos et al. 2007).

The aim of this paper was to investigate and develop characterizations of admissibility in the context of De Morgan algebras. A rule is admissible in a logic (understood as a consequence relation) if every substitution that makes each premise of the rule into a theorem of the logic also makes the conclusion into a theorem. Equivalently, a rule is admissible if it can be added to the logic without producing any new theorems. The admissible rules of classical propositional logic are also derivable (i.e., classical propositional logic is structurally complete), but this is not the case in general for non-classical logics (see Rybakov 1997; Ghilardi 1999, 2000; Iemhoff 2001; Jeřábek 2005, 2010a, b; Olson et al. 2008; Cintula and Metcalfe 2009, 2010). In algebra, rules correspond (roughly speaking) to quasiidentities and the admissible quasi-identities of a quasivariety may be understood as the quasi-identities that 
hold in the free algebra on countably infinitely many generators.

For algebraizable logics, admissible rules may be translated into admissible quasi-identities and vice versa. De Morgan algebras provide semantics for Belnap's fourvalued logic (which, note, has no theorems), but do not form the equivalent algebraic semantics for any algebraizable logic (see Font 1997 for details) and we therefore focus only on the algebraic notion. In particular, we give characterizations here of admissible quasi-identities for the classes of Kleene lattices $\mathrm{KL}$, Kleene algebras KA, De Morgan lattices DML, and De Morgan algebras DMA. For $\mathrm{KL}, \mathrm{KA}$, and DML, axiomatizations of the admissible quasi-identities make use of a single additional quasiidentity. However, in the case of DMA, we make use of not only a quasi-identity but also a universal formula. We conclude by giving a brief overview of recent admissibility and structural completeness results for some related classes of algebras.

\section{Admissibility in quasivarieties}

Let us begin by briefly recalling some basic notions from universal algebra, referring to Burris and Sankappanavar (1981) for further details. For a language $\mathcal{L}$, we denote the formula algebra over countably infinitely many variables by $\mathbf{F} \mathbf{m}_{\mathcal{L}}$ and let the metavariables $\varphi, \psi, \chi$ stand for arbitrary members of $\mathrm{Fm}_{\mathcal{L}}$ called $\mathcal{L}$-formulas. An $\mathcal{L}$-identity is a pair of $\mathcal{L}$-formulas, written $\varphi \approx \psi$, and we let the metavariables $\Sigma, \Delta$ stand for arbitrary finite sets of $\mathcal{L}$-identities. An $\mathcal{L}$ quasi-identity is identified with an ordered pair consisting of a finite set of $\mathcal{L}$-identities $\Sigma$ and a single $\mathcal{L}$-identity $\varphi \approx \psi$, written $\Sigma \Rightarrow \varphi \approx \psi$ (often dropping the external brackets in $\Sigma$ ). We denote sets of $\mathcal{L}$-quasi-identities using the metavariable $\Lambda$. As usual, if the language is clear from the context we may omit the prefix $\mathcal{L}$ when referring to these concepts.

Let $\mathcal{K}$ be a class of algebras of the same language $\mathcal{L}$ and let $\Sigma \cup\{\varphi \approx \psi\}$ be a finite set of $\mathcal{L}$-identities. We write $\Sigma \models_{\mathcal{K}} \varphi \approx \psi$ to denote that for every $\mathbf{A} \in \mathcal{K}$ and homomorphism $h: \mathbf{F m}_{\mathcal{L}} \rightarrow \mathbf{A}, \Sigma \subseteq \operatorname{ker} h$ implies $\varphi \approx \psi \in \operatorname{ker} h$. We abbreviate $\emptyset \models_{\mathcal{K}} \varphi \approx \psi$ by $\models_{\mathcal{K}} \varphi \approx \psi$, and $\Sigma \models_{\{\mathbf{A}\}}$ $\varphi \approx \psi$ by $\Sigma \models_{\mathbf{A}} \varphi \approx \psi$, saying in the latter case that the quasi-identity $\Sigma \Rightarrow \varphi \approx \psi$ "holds in" the algebra $\mathbf{A} . \mathcal{K}$ is said to be a quasivariety if there exists a set of $\mathcal{L}$-quasiidentities $\Lambda$ such that $\mathbf{A} \in \mathcal{K}$ if and only if (henceforth, iff) all quasi-identities in $\Lambda$ hold in $\mathbf{A}$. If there exists such a $\Lambda$ consisting only of identities, then $\mathcal{K}$ is called a variety. The variety $\mathbb{V}(\mathcal{K})$ and quasivariety $\mathbb{Q}(\mathcal{K})$ generated by $\mathcal{K}$ are, respectively, the smallest variety and quasivariety containing $\mathcal{K}$.
Now let $\mathcal{Q}$ be a quasivariety for a language $\mathcal{L}$. An $\mathcal{L}$-quasi-identity $\Sigma \Rightarrow \varphi \approx \psi$ will be called admissible in $\mathcal{Q}$, if for every homomorphism $\sigma: \mathbf{F m}_{\mathcal{L}} \rightarrow \mathbf{F m}_{\mathcal{L}}$ :

$\models_{\mathcal{Q}} \sigma\left(\varphi^{\prime}\right) \approx \sigma\left(\psi^{\prime}\right)$ for all $\varphi^{\prime} \approx \psi^{\prime} \in \Sigma$ implies

$\models_{\mathcal{Q}} \sigma(\varphi) \approx \sigma(\psi)$.

In fact, quasi-identities admissible in $\mathcal{Q}$ are simply the quasi-identities that hold in the free algebra on countably infinitely many generators of $\mathcal{Q}$, denoted $\mathbf{F}_{\mathcal{Q}}$. To establish this well-known but crucial fact, we make use of the canonical homomorphism $h_{\mathcal{Q}}: \mathbf{F m}_{\mathcal{L}} \rightarrow \mathbf{F}_{\mathcal{Q}}$ that maps each formula to its equivalence class in $\mathbf{F}_{\mathcal{Q}}$, recalling (see Burris and Sankappanavar 1981 for details) that for each $\mathcal{L}$ identity $\varphi \approx \psi$ :

$\models_{\mathcal{Q}} \varphi \approx \psi \quad$ iff $\quad \models_{\mathbf{F}_{\mathcal{Q}}} \varphi \approx \psi$ iff $\quad h_{\mathcal{Q}}(\varphi)=h_{\mathcal{Q}}(\psi)$.

Lemma 1 Given a quasivariety $\mathcal{Q}$ for a language $\mathcal{L}$ and $\mathcal{L}$-quasi-identity $\Sigma \Rightarrow \varphi \approx \psi:$

$\Sigma \Rightarrow \varphi \approx \psi$ is admissible in $\mathcal{Q}$ iff $\Sigma \models_{\mathbf{F}_{\mathcal{Q}}} \varphi \approx \psi$.

Proof Suppose that $\Sigma \Rightarrow \varphi \approx \psi$ is admissible in $\mathcal{Q}$ and let $g: \mathbf{F m}_{\mathcal{L}} \rightarrow \mathbf{F}_{\mathcal{Q}}$ be a homomorphism such that $\Sigma \subseteq \operatorname{ker} g$. We define a map $\sigma$ that sends each variable $x$ to a member of the equivalence class $g(x)$. By the universal mapping property for $\mathbf{F m}_{\mathcal{L}}$, this extends to a homomorphism $\sigma: \mathbf{F m}_{\mathcal{L}} \rightarrow \mathbf{F} \mathbf{m}_{\mathcal{L}}$. But since $h_{\mathcal{Q}}(\sigma(x))=g(x)$ for each variable $x$, we obtain $h_{\mathcal{Q}} \circ \sigma=g$. But then $\Sigma \subseteq \operatorname{ker}\left(h_{\mathcal{Q}} \circ\right.$ $\sigma)$, so for each $\varphi^{\prime} \approx \psi^{\prime} \in \Sigma$, we have $h_{\mathcal{Q}}\left(\sigma\left(\varphi^{\prime}\right)\right)=$ $h_{\mathcal{Q}}\left(\sigma\left(\psi^{\prime}\right)\right)$ and therefore $\models_{\mathcal{Q}} \sigma\left(\varphi^{\prime}\right) \approx \sigma\left(\psi^{\prime}\right)$. Hence by assumption, $\models_{\mathcal{Q}} \sigma(\varphi) \approx \sigma(\psi)$, and $g(\varphi)=h_{\mathcal{Q}}(\sigma(\varphi))=$ $h_{\mathcal{Q}}(\sigma(\psi))=g(\psi)$ as required.

Suppose now that $\Sigma \models_{\mathbf{F}_{\mathcal{Q}}} \varphi \approx \psi$ and let $\sigma: \mathbf{F m}_{\mathcal{L}} \rightarrow \mathbf{F m}_{\mathcal{L}}$ be a homomorphism such that for each $\varphi^{\prime} \approx \psi^{\prime} \in \Sigma, \models_{\mathcal{Q}}$ $\sigma\left(\varphi^{\prime}\right) \approx \sigma\left(\psi^{\prime}\right)$ and hence $h_{\mathcal{Q}}\left(\sigma\left(\varphi^{\prime}\right)\right)=h_{\mathcal{Q}}\left(\sigma\left(\psi^{\prime}\right)\right)$. By assumption, $h_{\mathcal{Q}}(\sigma(\varphi))=h_{\mathcal{Q}}(\sigma(\psi))$. Hence $\models_{\mathcal{Q}} \sigma(\varphi) \approx$ $\sigma(\psi)$ as required.

Example 1 Consider the variety of abelian groups. The quasi-identities $(1 \leq n \in \mathbb{N})$

$\underbrace{x+\cdots+x}_{n} \approx 0 \Rightarrow x \approx 0$

(which define the quasivariety of torsion-free abelian groups) hold in all free abelian groups and are hence admissible in the variety, but do not hold in all abelian groups.

A quasivariety $\mathcal{Q}$ is called structurally complete if each of its proper subquasivarieties generates a proper subvariety of $\mathbb{V}(\mathcal{Q})$, i.e., for each quasivariety $\mathcal{Q}^{\prime} \subset \mathcal{Q}$, we have $\mathbb{V}\left(\mathcal{Q}^{\prime}\right) \subset \mathbb{V}(\mathcal{Q})$. Equivalently (proved by Bergman 1991, Proposition 2.3), $\mathcal{Q}$ is structurally complete iff $\mathcal{Q}=\mathbb{Q}\left(\mathbf{F}_{\mathcal{Q}}\right)$. Hence, combining with Lemma 1: 
Corollary 1 The following are equivalent for any quasivariety $\mathcal{Q}$ for a language $\mathcal{L}$ :
(a) $\mathcal{Q}$ is structurally complete
(b) $\mathcal{Q}=\mathbb{Q}\left(\mathbf{F}_{\mathcal{Q}}\right)$
(c) An $\mathcal{L}$-quasi-identity $\Sigma \Rightarrow \varphi \approx \psi$ is admissible in $\mathcal{Q}$ iff $\Sigma \models_{\mathcal{Q}} \varphi \approx \psi$.

This corollary supplies a method for establishing structural completeness for quasivarieties. A quasivariety $\mathcal{Q}$ is structurally complete if each member of a class of algebras generating $\mathcal{Q}$ as a quasivariety can be embedded into $\mathbf{F}_{\mathcal{Q}}$, since then any quasi-identity failing in one of the generating algebras must fail in $\mathbf{F}_{\mathcal{Q}}$. More precisely

Lemma 2 Cintula and Metcalfe (2009, Theorem 3.3) Let $\mathcal{Q}=\mathbb{Q}(\mathcal{K})$ be the quasivariety generated by a class of algebras $\mathcal{K}$ of the same language $\mathcal{L}$ and suppose that for each $\mathbf{A} \in \mathcal{K}$, there is a map $g^{\mathbf{A}}: A \rightarrow \mathrm{Fm}_{\mathcal{L}}$ such that $h_{\mathcal{Q}} \circ g^{\mathbf{A}}$ embeds $\mathbf{A}$ into $\mathbf{F}_{\mathcal{Q}}$. Then $\mathcal{Q}$ is structurally complete.

Example 2 Consider the variety BA of Boolean algebras, generated as a quasivariety by the algebra $\mathbf{2}=$ $\langle\{0,1\}, \wedge, \vee, \neg, 0,1\rangle$. Define $g(0)=\perp$ and $g(1)=\top$. Then $h_{\mathrm{BA}} \circ g$ embeds 2 into $\mathbf{F}_{\mathrm{BA}}$. So BA is structurally complete.

Let $\mathcal{Q}$ and $\mathcal{Q}^{\prime}$ be quasivarieties for a language $\mathcal{L}$ and let $\Lambda$ be a set of $\mathcal{L}$-quasi-identities. Suppose that $\mathbf{A} \in \mathcal{Q}^{\prime}$ iff both $\mathbf{A} \in \mathcal{Q}$ and each quasi-identity in $\Lambda$ holds in $\mathbf{A}$. Then $\Lambda$ is said to axiomatize $\mathcal{Q}^{\prime}$ relative to $\mathcal{Q}$. In particular, if $\mathbb{Q}\left(\mathbf{F}_{\mathcal{Q}}\right)$ is axiomatized by $\Lambda$ relative to $\mathcal{Q}$, then we call $\Lambda$ a basis for the admissible quasi-identities of $\mathcal{Q}$.

Since $\mathbb{Q}\left(\mathbf{F}_{\mathcal{Q}}\right) \subseteq \mathcal{Q}$ for any quasivariety $\mathcal{Q}$, finding a basis for the admissible quasi-identities of $\mathcal{Q}$ essentially involves finding a set of quasi-identities that are admissible in $\mathcal{Q}$ and that axiomatize a structurally complete quasivariety relative to $\mathcal{Q}$. More precisely

Lemma 3 Let $\mathcal{Q}$ and $\mathcal{Q}^{\prime}$ be quasivarieties for a language $\mathcal{L}$ and let $\Lambda$ be a set of $\mathcal{L}$-quasi-identities axiomatizing $\mathcal{Q}^{\prime}$ relative to $\mathcal{Q}$. Suppose that $\mathcal{Q}^{\prime}$ is structurally complete and that each quasi-identity in $\Lambda$ is admissible in $\mathcal{Q}$. Then $\Lambda$ is a basis for the admissible quasi-identities of $\mathcal{Q}$.

Proof It suffices to show that $\mathcal{Q}^{\prime}=\mathbb{Q}\left(\mathbf{F}_{\mathcal{Q}}\right)$. If each quasiidentity in $\Lambda$ is admissible in $\mathcal{Q}$, then by Lemma 1 , each quasi-identity in $\Lambda$ holds in $\mathbf{F}_{\mathcal{Q}}$. Hence $\mathbf{F}_{\mathcal{Q}} \in \mathcal{Q}^{\prime}$ and $\mathbb{Q}\left(\mathbf{F}_{\mathcal{Q}}\right) \subseteq \mathcal{Q}^{\prime}$. Suppose for a contradiction that $\mathbb{Q}\left(\mathbf{F}_{\mathcal{Q}}\right) \subset \mathcal{Q}^{\prime}$. Since $\mathcal{Q}^{\prime}$ is structurally complete, $\mathbb{V}(\mathcal{Q})=\mathbb{V}\left(\mathbf{F}_{\mathcal{Q}}\right)=$ $\mathbb{V}\left(\mathbb{Q}\left(\mathbf{F}_{\mathcal{Q}}\right)\right) \subset \mathbb{V}\left(\mathcal{Q}^{\prime}\right)$ (recalling that $\mathbb{V}(\mathcal{Q})=\mathbb{V}\left(\mathbf{F}_{\mathcal{Q}}\right)$ follows from Burris and Sankappanavar (1981, Theorem 11.4). But $\mathcal{Q}^{\prime} \subseteq \mathcal{Q}$, so $\mathbb{V}\left(\mathcal{Q}^{\prime}\right) \subseteq \mathbb{V}(\mathcal{Q})$, a contradiction.
For convenience, in the remainder of this paper, we will use the symbols $\mathcal{L}^{l}$ and $\mathcal{L}^{b}$ to denote, respectively, the languages of De Morgan lattices and De Morgan algebras.

\section{Kleene algebras}

Recall from the introduction that a Kleene algebra is a De Morgan algebra satisfying

$x \wedge \neg x \leq y \vee \neg y$

and that the variety of Kleene algebras is denoted by KA. The following finite algebras are particularly useful members of this variety (where $1 \leq m \in \mathbb{N}$ ):

$$
\begin{aligned}
\mathbf{C}_{\mathbf{2} \mathbf{m}}=\langle\{ & -m,-m+1, \ldots,-1,1, \ldots, m-1, m\}, \\
& \min , \max ,-,-m, m\rangle \\
\mathbf{C}_{\mathbf{2} \mathbf{m}+\mathbf{1}}=\langle\{ & \langle-m,-m+1, \ldots,-1,0,1, \ldots, m-1, m\}, \\
& \quad \min , \max ,-,-m, m\rangle .
\end{aligned}
$$

The "fuzzy algebra" $\langle[0,1], \min , \max , 1-x, 0,1\rangle$ and also each $\mathbf{C}_{\mathbf{n}}$ for any odd $n \geq 3$, generates $\mathrm{KA}$ as a quasivariety. In particular, $K A=\mathbb{Q}\left(\mathbf{C}_{\mathbf{3}}\right)$ (see, e.g., Kalman 1958; Pynko 1999).

Now consider the quasi-identity

$x \approx \neg x \Rightarrow x \approx y$.

We have $\{x \approx \neg x\} \forall_{\mathbf{C}_{3}} x \approx y:$ just consider an evaluation sending $x$ to 0 and $y$ to 1 . But there is no formula $\varphi$ such that $\varphi \approx \neg \varphi$ holds in all Kleene algebras (or indeed, in all Boolean algebras). So the quasi-identity (1) is admissible and by Corollary $1, \mathrm{KA}$ is not structurally complete.

However, the proper subquasivariety of KA generated by $\mathbf{C}_{\mathbf{n}}$ for any even $n \geq 4$ is structurally complete. In particular

Lemma $4 \mathbb{Q}\left(\mathbf{C}_{\mathbf{4}}\right)$ is structurally complete.

Proof By Lemma 2, it suffices to find a map $g: \mathbf{C}_{\mathbf{4}} \rightarrow \mathbf{F m}_{\mathcal{L}^{\mathrm{b}}}$ such that $h_{\mathbb{Q}\left(\mathbf{C}_{4}\right)} \circ g$ embeds $\mathbf{C}_{\mathbf{4}}$ into $\mathbf{F}_{\mathbb{Q}\left(\mathbf{C}_{4}\right)}$. Define $g: \mathbf{C}_{\mathbf{4}} \rightarrow \mathbf{F m}_{\mathcal{L}^{b}}$ by $g(1)=x \vee \neg x, g(-1)=x \wedge \neg x$, $g(2)=\top$, and $g(-2)=\perp$. Then $h_{\mathbb{Q}\left(\mathbf{C}_{4}\right)} \circ g$ preserves the operations of $\mathbf{C}_{4}$. E.g., for all $a \in C_{4}$,

$\left(h_{\mathbb{Q}\left(\mathbf{C}_{4}\right)} \circ g\right)(\neg a)=\neg\left(h_{\mathbb{Q}\left(\mathbf{C}_{4}\right)} \circ g\right)(a)$

follows from the fact that $\models_{\mathbf{C}_{4}} \neg \perp \approx \top, \models_{\mathbf{C}_{4}} \neg \top \approx \perp, \models_{\mathbf{C}_{4}}$ $\neg(x \wedge \neg x) \approx x \vee \neg x$, and $\models_{\mathbf{C}_{4}} \neg(x \vee \neg x) \approx x \wedge \neg x$. Moreover, $h_{\mathbb{Q}\left(\mathbf{C}_{4}\right)} \circ g$ is one-to-one, since $\forall_{\mathbf{C}_{4}} \varphi \approx \psi$ for any distinct $\varphi$ and $\psi$ from $x \vee \neg x, x \wedge \neg x, \top$, and $\perp$.

Following almost exactly the corresponding proof of Pynko (1999, Proposition 4.7) for Kleene lattices (see also Gaitán and Perea 2004, p. 239), $\mathbb{Q}\left(\mathbf{C}_{4}\right)$ is axiomatized relative to $\mathrm{KA}$ by the quasi-identity 
$\neg x \leq x, x \wedge \neg y \leq \neg x \vee y \Rightarrow \neg y \leq y$.

Hence we obtain

Theorem $1\{(2)\}$ is a basis for the admissible quasiidentities of $\mathrm{KA}$.

Proof $\mathbb{Q}\left(\mathbf{C}_{\mathbf{4}}\right)$ is structurally complete (Lemma 4) and axiomatized relative to KA by $\{(2)\}$ (see Pynko 1999, Proposition 4.7). Moreover, $\mathbf{C}_{\mathbf{3}}$ is a homomorphic image of $\mathbf{C}_{\mathbf{4}}$, so $\mathbb{V}\left(\mathbf{C}_{\mathbf{4}}\right)=\mathbb{V}\left(\mathbf{C}_{\mathbf{3}}\right)=\mathrm{KA}$. Hence, since (2) holds in $\mathbf{C}_{\mathbf{4}}$, it is admissible in $\mathrm{KA}$, and the result follows by Lemma 3.

Note that the quasi-identity (1) does not provide a basis for the admissible quasi-identities of KA. In fact, it axiomatizes the quasivariety $\mathbb{Q}\left(\mathbf{C}_{\mathbf{3}} \times \mathbf{C}_{\mathbf{2}}\right)$ relative to $\mathrm{KA}$ (see Pynko 1999, Proposition 4.5). We remark also that by almost exactly the same reasoning, we can show that $\{(2)\}$ provides a basis for the admissible quasi-identities of the class $\mathrm{KL}$ of Kleene lattices. The only difference lies in the proof of Lemma 4 that the quasivariety of Kleene lattices generated by the four-element chain is structurally complete: in this case we simply change the mapping $g$ to $g(1)=x \vee \neg x, g(-1)=x \wedge \neg x, g(2)=(x \vee \neg x) \vee y, \quad$ and $g(-2)=(x \wedge \neg x) \wedge \neg y$.

\section{De Morgan algebras}

The class DMA of De Morgan algebras is generated as a quasivariety by the four-valued diamond algebra

$\mathbf{D}_{\mathbf{4}}=\langle\{\perp, a, b, \top\}, \wedge, \vee, \neg, \perp, \top\rangle$

where $\langle\{\perp, a, b, \top\}, \wedge, \vee, \perp, \top\rangle$ is the diamond bounded lattice of Fig. 1 and $\neg$ is defined by $\neg \perp=T, \neg \top=$ $\perp, \neg a=a$, and $\neg b=b$. That is, $\mathrm{DMA}=\mathbb{Q}\left(\mathbf{D}_{4}\right)$ (see Kalman 1958). However, to obtain a characterization of the admissible quasi-identities of DMA, it will be helpful to first consider the (easier) case of the class DML of De Morgan lattices. Let us write $\mathbf{A}^{\mathbf{L}}$ to denote the De Morgan lattice reduct of a De Morgan algebra $\mathbf{A}$, noting that $\mathrm{DML}=\mathbb{Q}\left(\mathbf{D}_{4}^{\mathbf{L}}\right)$.

The finite lattice of quasivarieties of DML has been completely characterized by Pynko (1999) and consists of just seven non-trivial quasivarieties of De Morgan lattices related as described in Fig. 2. Recall that a quasivariety $\mathcal{Q}$ is structurally complete if every proper subquasivariety of $\mathcal{Q}$ generates a proper subvariety of $\mathbb{V}(\mathcal{Q})$. The only nontrivial varieties of De Morgan lattices are $B L=\mathbb{Q}\left(\mathbf{C}_{2}^{\mathbf{L}}\right)$, $\mathrm{KL}=\mathbb{Q}\left(\mathbf{C}_{\mathbf{3}}^{\mathbf{L}}\right)$, and $\mathrm{DML}=\mathbb{Q}\left(\mathbf{D}_{\mathbf{4}}^{\mathbf{L}}\right)$. Hence by inspection of the subquasivariety lattice, the only non-trivial structurally complete subquasivarieties of $\mathrm{DML}$ are $\mathrm{BL}=\mathbb{Q}\left(\mathbf{C}_{\mathbf{2}}^{\mathbf{L}}\right)$, $\mathbb{Q}\left(\mathbf{C}_{\mathbf{4}}^{\mathbf{L}}\right)$, and $\mathbb{Q}\left(\mathbf{D}_{\mathbf{4 2}}^{\mathbf{L}}\right)$ where $\mathbf{D}_{\mathbf{4 2}}$ is defined as the direct
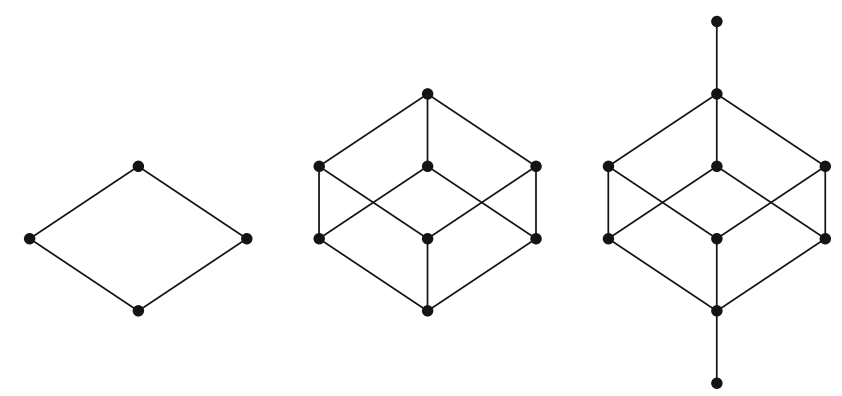

Fig. 1 The De Morgan algebras $\mathbf{D}_{\mathbf{4}}, \mathbf{D}_{\mathbf{4 2}}$, and $\overline{\mathbf{D}_{\mathbf{4 2}}}$

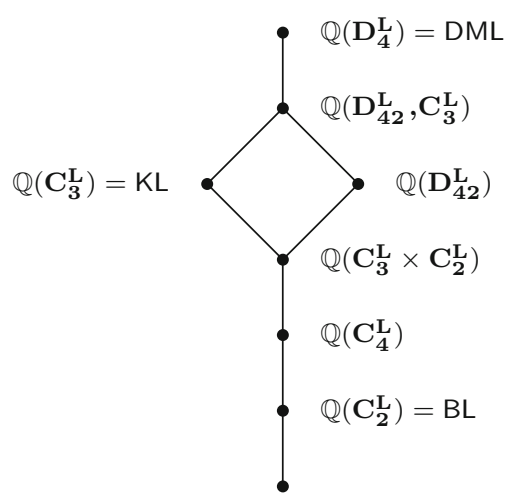

Fig. 2 Subquasivarieties of DML

product $\mathbf{D}_{\mathbf{4}} \times \mathbf{C}_{\mathbf{2}}$ (see Fig. 1). It follows in particular that the admissible quasi-identities of DML must be precisely those holding in $\mathbb{Q}\left(\mathbf{D}_{\mathbf{4 2}}^{\mathbf{L}}\right)$. Moreover, Pynko (1999, Proposition 4.2) has shown that $\mathbb{Q}\left(\mathbf{D}_{\mathbf{4 2}}^{\mathbf{L}}\right)$ is axiomatized relative to DML by the quasi-identity (1). Hence $\{(1)\}$ is a basis for the admissible quasi-identities of DML. Here, however, we give a more direct proof that avoids the need for a full investigation of the subquasivariety lattice.

Lemma $5 \mathbb{Q}\left(\mathbf{D}_{\mathbf{4 2}}^{\mathbf{L}}\right)$ is structurally complete.

Proof By Lemma 2, it suffices to give an embedding of $\mathbf{D}_{\mathbf{4 2}}^{\mathbf{L}}$ into $\mathbf{F}_{\mathbb{Q}}\left(\mathbf{D}_{\mathbf{4 2}}^{\mathbf{L}}\right)$. We first define for distinct variables $x_{1}$ and $x_{2}$,

$\varphi=x_{1} \wedge \neg x_{1} \quad$ and $\quad \psi=x_{2} \vee \neg x_{2}$,

and note that $\varphi<\neg \varphi$ and $\neg \psi<\psi$ in $\mathbf{F}_{\mathbb{Q}\left(\mathbf{D}_{42}^{\mathbf{L}}\right)}$. Now we define $e: \mathbf{D}_{\mathbf{4 2}}^{\mathbf{L}} \rightarrow \mathbf{F}_{\mathbb{Q}\left(\mathbf{D}_{42}^{\mathbf{L}}\right)}$ by $e=h_{\mathbb{Q}\left(\mathbf{D}_{\mathbf{4 2}}^{\mathbf{L}}\right)} \circ g$ where

$$
\begin{array}{ll}
g(\perp,-1)=(\varphi \wedge \psi) \vee(\neg \varphi \wedge \neg \psi) & g(\perp, 1)=\psi \wedge \neg \varphi \\
g(a,-1)=\varphi \vee(\neg \varphi \wedge \neg \psi) & g(a, 1)=(\varphi \vee \psi) \wedge \neg \varphi \\
g(b,-1)=(\varphi \wedge \psi) \vee \neg \psi & g(b, 1)=\psi \wedge(\neg \varphi \vee \neg \psi) \\
g(\top,-1)=\varphi \vee \neg \psi & g(T, 1)=(\varphi \vee \psi) \wedge(\neg \varphi \vee \neg \psi) .
\end{array}
$$

It is straightforward to check that none of these formulas are equivalent to each other in $\mathbf{F}_{\mathbb{Q}\left(\mathbf{D}_{\mathbf{4 2}}^{\mathrm{L}}\right)}$ and hence that the mapping is one-to-one. It remains to check that $e$ is a homomorphism. First note that $\neg$ is preserved by the 
mapping; e.g., $e(\neg(a, 1))=e(a,-1)=\neg e(a, 1)$ follows from the fact that

$x \vee(\neg x \wedge \neg y) \approx \neg((x \vee y) \wedge \neg x)$

holds in $\mathbf{F}_{\mathbb{Q}\left(\mathbf{D}_{\mathbf{4 2}}^{\mathbf{L}}\right)}$. To see that $\wedge$ is preserved by the mapping (the case of $\vee$ is dual), we note that

$(x \wedge y) \vee(\neg x \wedge \neg y)<x \vee(\neg x \wedge \neg y)<x \vee \neg y$

$(x \wedge y) \vee(\neg x \wedge \neg y)<(x \wedge y) \vee \neg y<x \vee \neg y$

$y \wedge \neg x<(x \vee y) \wedge \neg x<(x \vee y) \wedge(\neg x \vee \neg y)$

$y \wedge \neg x<y \wedge(\neg x \vee \neg y)<(x \vee y) \wedge(\neg x \vee \neg y)$

hold in $\mathbf{F}_{\mathbb{Q}}\left(\mathbf{D}_{\mathbf{4 2}}^{\mathbf{L}}\right)$. Moreover, using the fact that $\varphi<\neg \varphi$ and $\neg \psi<\psi$ in $\mathbf{F}_{\mathbb{Q}}\left(\mathbf{D}_{\mathbf{4 2}}^{\mathbf{L}}\right)$

$(\varphi \wedge \psi) \vee(\neg \varphi \wedge \neg \psi)<\psi \wedge \neg \varphi$

$\varphi \vee(\neg \varphi \wedge \neg \psi)<(\varphi \vee \psi) \wedge \neg \varphi$

$(\varphi \wedge \psi) \vee \neg \psi<\psi \wedge(\neg \varphi \vee \neg \psi)$

$\varphi \vee \neg \psi<(\varphi \vee \psi) \wedge(\neg \varphi \vee \neg \psi)$

hold in $\mathbf{F}_{\mathbb{Q}}\left(\mathbf{D}_{\mathbf{4 2}}^{\mathbf{L}}\right)$, as do the following

$$
\begin{aligned}
&(\varphi \vee \neg \psi) \wedge(\psi \wedge \neg \varphi) \approx(\varphi \wedge(\psi \wedge \neg \varphi)) \vee(\neg \psi \wedge(\psi \\
&\wedge \neg \varphi)) \\
& \approx(\varphi \wedge \psi) \vee(\neg \varphi \wedge \neg \psi) \\
&(\varphi \vee(\neg \varphi \wedge \neg \psi)) \wedge(\psi \wedge(\neg \varphi \vee \neg \psi))(\varphi \wedge) \\
& \approx(\varphi \wedge \psi) \vee(\neg \varphi \wedge \neg \psi) \\
&((\varphi \wedge \psi) \vee \neg \psi) \wedge((\varphi \vee \psi) \wedge \neg \varphi) \\
& \quad \approx(\varphi \wedge \psi) \vee(\neg \varphi \wedge \neg \psi)
\end{aligned}
$$

so $\wedge$ is preserved by $e$.

Theorem $2\{(1)\}$ is a basis for the admissible quasiidentities of $\mathrm{DML}$.

Proof (1) is admissible in DML and the quasivariety $\mathbb{Q}\left(\mathbf{D}_{\mathbf{4 2}}^{\mathbf{L}}\right)$ is structurally complete (Lemma 5) and axiomatized relative to DML by $\{(1)\}$ (Pynko 1999, Proposition 4.2). Hence the result follows by Lemma 3.

We now turn our attention to De Morgan algebras. Here the picture is not so clear since the quasivariety lattice is infinite (see Gaitán and Perea 2004). In particular, unlike the case of DML, the quasi-identity (1) does not provide a basis for the admissible quasi-identities of DMA. It follows from results of Pynko (1999) that $\{(1)\}$ axiomatizes the quasivariety $\mathbb{Q}\left(\mathbf{D}_{\mathbf{4 2}}\right)$ relative to DMA. However, the quasiidentity

$(x \wedge \neg x) \vee y \approx \top \Rightarrow y \approx \top$

is admissible in DMA but does not hold in the De Morgan algebra $\mathbf{D}_{\mathbf{4 2}}$. So $\{(1)\}$ cannot suffice as a basis for the admissible quasi-identities of DMA.
Let us consider instead the De Morgan algebra $\overline{\mathbf{D}_{\mathbf{4 2}}}$ obtained from $\mathbf{D}_{\mathbf{4 2}}$ by adding an extra top element $T$ and bottom element $\perp$ (see Fig. 1). Note that $\mathbf{D}_{\mathbf{4}}$ is a homomorphic image of $\overline{\mathbf{D}_{\mathbf{4 2}}}$ under the composition of $f: \overline{\mathbf{D}_{\mathbf{4 2}}} \rightarrow$ $\mathbf{D}_{\mathbf{4 2}}, f(\top)=(\top, 1), f(\perp)=(\perp, 0), f((x, y))=(x, y)$ for all $(x, y) \notin\{\perp, \top\}$ and the projection $p: \mathbf{D}_{\mathbf{4 2}} \rightarrow \mathbf{D}_{\mathbf{4}}, p(x, y)=$ $x$. Hence $\mathbb{V}\left(\mathbb{Q}\left(\overline{\mathbf{D}_{\mathbf{4 2}}}\right)\right)=$ DMA. Moreover,

Lemma $6 \mathbb{Q}\left(\overline{\mathbf{D}_{42}}\right)$ is structurally complete.

Proof We extend the embedding given in the proof of Lemma 5 by defining

$g(\perp)=\perp \quad$ and $\quad g(\top)=\top$

to obtain an embedding of $\overline{\mathbf{D}_{\mathbf{4 2}}}$ into $\mathbf{F}_{\mathbb{Q}}\left(\overline{\mathbf{D}_{\mathbf{4 2}}}\right)$. We simply note additionally that $\neg \top \approx \perp, \neg \perp \approx \top, \top \wedge x \approx x, \top \vee$ $x \approx \top, \perp \wedge x \approx \perp$, and $\perp \vee x \approx x$ all hold in $\mathbf{F}_{\mathbb{Q}}\left(\overline{\mathbf{D}_{\mathbf{4 2}}}\right)$.

It follows that the admissible quasi-identities of DMA consist of those quasi-identities that hold in $\mathbb{Q}\left(\overline{\mathbf{D}_{\mathbf{4 2}}}\right)$. However, unlike the cases of Kleene algebras and De Morgan lattices, we have been unable to find an axiomatization of this quasivariety using just quasi-identities. Instead, we make use also of a universal formula. More precisely, for a language $\mathcal{L}$, we identify universal formulas consisting of an ordered pair of finite sets $\Sigma, \Delta$ of $\mathcal{L}$-formulas, written $\Sigma \Rightarrow \Delta$ (often dropping brackets). For a class of algebras $\mathcal{K}$ for $\mathcal{L}$, we write $\Sigma \models_{\mathcal{K}} \Delta$ to denote that for every $\mathbf{A} \in \mathcal{K}$ and homomorphism $h: \mathbf{F m}_{\mathcal{L}} \rightarrow \mathbf{A}, \Sigma \subseteq$ ker $h$ implies $\Delta \cap \operatorname{ker} h \neq \emptyset$. As for quasi-identities, we drop brackets when considering just one algebra and say that the universal formula "holds in" this algebra.

Observe that the following universal formula holds in $\mathbf{D}_{\mathbf{4 2}}^{-}$and hence also in $\mathbf{F}_{\mathrm{DMA}}$ :

$x \vee y \approx \top \Rightarrow x \approx \top, y \approx \top$.

Let us define $\mathrm{DMA}^{*}$ to be the class of all De Morgan algebras A such that the quasi-identity (1) and the universal formula (3) both hold in A. We will show that a quasiidentity is admissible in DMA iff it holds in all members of DMA*. The main idea of the proof will be to reduce the question of the admissibility of a quasi-identity in DMA to the question of the admissibility of certain quasi-identities in DML. The following lemma, proved by an easy induction on $c(\varphi)$, the number of occurrences of connectives $\wedge, \vee$, and $\neg$ in a formula $\varphi$, will be useful in this respect.

Lemma 7 For any $\varphi \in \mathrm{Fm}_{\mathcal{L}^{b}}$, one of the following holds:

1. $\models$ DMA $\varphi \approx \perp$

2. $\models$ DMA $\varphi \approx \top$

3. $\models_{\mathrm{DMA}} \varphi \approx \psi$ for some $\psi \in \mathrm{Fm}_{\mathcal{L}^{l}}$ with $c(\psi) \leq c(\varphi)$.

Let us say that an $\mathcal{L}^{b}$-identity $\varphi \approx \psi$ is in normal form if $\varphi$ and $\psi$ are either $\perp, \top$, or members of $\mathrm{Fm}_{\mathcal{L}^{l}}$. 
Theorem 3 For any $\mathcal{L}^{b}$-quasi-identity $\Sigma \Rightarrow \varphi \approx \psi$ : $\Sigma \Rightarrow \varphi \approx \psi$ is admissible in DMA iff $\Sigma \models_{\mathrm{DMA}^{*}} \varphi \approx \psi$.

Proof Suppose first that $\Sigma \models \mathrm{DMA}^{*} \varphi \approx \psi$. Both the quasiidentity (1) and the universal formula (3) hold in $\mathbf{F}_{\mathrm{DMA}}$, so $\mathbf{F}_{\mathrm{DMA}} \in \mathrm{DMA}^{*}$. Hence $\Sigma \models_{\mathbf{F}_{\mathrm{DMA}}} \varphi \approx \psi$ and by Lemma 1 , $\Sigma \Rightarrow \varphi \approx \psi$ is admissible in DMA. For the other direction, it suffices, using Lemmas 7 and 1, to prove the following:

For any finite set $\Sigma \cup\{\varphi \approx \psi\}$ of $\mathcal{L}^{b}$-identities in normal form:

$\Sigma \models_{\mathbf{F}_{\mathrm{DMA}}} \varphi \approx \psi \quad$ implies $\quad \Sigma \models_{\mathrm{DMA}^{*}} \varphi \approx \psi$.

Let $c(\Sigma)$ be the number of occurrences of connectives $\wedge, \vee$, and $\neg$ in $\Sigma$ and let $s(\Sigma)$ be the number of identities in $\Sigma$ containing $\perp$ or $T$. We prove $(\star)$ by induction on the lexicographically ordered pair $\langle c(\Sigma), s(\Sigma)\rangle$. The idea is to successively eliminate occurrences of $\perp$ and $T$ in $\Sigma$ by reducing $\langle c(\Sigma), s(\Sigma)\rangle$.

Base case. Suppose that there are no occurrences of $\perp$ and $\top$ in $\Sigma$, i.e., $s(\Sigma)=0$. If $\varphi=\psi$ or $\{\varphi, \psi\} \subseteq\{\perp, \top\}$, then we are done. Moreover, if $\varphi \in \operatorname{Fm}_{\mathcal{L}^{l}}$ and $\psi \in\{\perp, \top\}$, then $\Sigma \not \forall_{\mathbf{F}_{\mathrm{DMA}}} \varphi \approx \psi$ : just consider a homomorphism from $\mathbf{F m}_{\mathcal{L}^{\mathbf{b}}}$ to $\mathbf{D}_{\mathbf{4}}$ that maps all the variables to $a$. Finally, consider $\varphi, \psi \in \mathrm{Fm}_{\mathcal{L}^{l}}$. Suppose that $\Sigma \models_{\mathbf{F}_{\mathrm{DMA}}} \varphi \approx \psi$. By Lemma $1, \Sigma \Rightarrow \varphi \approx \psi$ is admissible in DMA. But for any $\varphi^{\prime}, \psi^{\prime} \in \mathrm{Fm}_{\mathcal{L}^{l}}$, we have $\models_{\mathrm{DMA}} \varphi^{\prime} \approx \psi^{\prime}$ iff $\models_{\mathbf{D}_{\mathbf{4}}} \varphi^{\prime} \approx \psi^{\prime}$ iff $\models_{\mathbf{D}_{4}^{\mathbf{L}}} \varphi^{\prime} \approx \psi^{\prime}$ iff $\models_{\mathrm{DML}} \varphi^{\prime} \approx \psi^{\prime}$. So $\Sigma \Rightarrow \varphi \approx \psi$ is admissible in DML. Hence by Theorem $2, \Sigma \Rightarrow \varphi \approx \psi$ holds in $\mathbb{Q}\left(\mathbf{D}_{\mathbf{4 2}}^{\mathbf{L}}\right)$. But every De Morgan algebra in $\mathrm{DMA}^{*}$ is also (ignoring $\perp$ and $T$ in the language) a De Morgan lattice in $\mathbb{Q}\left(\mathbf{D}_{\mathbf{4 2}}^{\mathbf{L}}\right)$, so $\Sigma \models$ DMA* $^{*} \varphi \approx \psi$.

Inductive step. Given $\Sigma$, suppose that $(\star)$ holds for all $\Delta$ such that $\langle c(\Delta), s(\Delta)\rangle\langle\langle c(\Sigma), s(\Sigma)\rangle$. We use $A \sqcup B$ to denote the disjoint union of two sets $A$ and $B$, i.e., $A \cap B=$ $\emptyset$. Consider the following cases:

- $\Sigma=\Delta \sqcup\{\perp \approx \top\}$. Then ( $\star$ ) clearly holds since $\Sigma \models$ DMA $^{*} \varphi \approx \psi$.

- $\Sigma=\Delta \sqcup\{\chi \approx \chi\}$. Then $\Sigma \models_{\mathbf{F}_{\mathrm{DMA}}} \varphi \approx \psi$ implies $\Delta \models_{\mathbf{F}_{\mathrm{DMA}}} \varphi \approx \psi$ and, by the induction hypothesis, $\Delta \models$ DMA $^{*} \varphi \approx \psi$. So $\Delta \sqcup\{\chi \approx \chi\} \models$ DMA $^{*} \varphi \approx \psi \quad$ as required.

- $\quad \Sigma=\Delta \sqcup\left\{\chi_{1} \vee \chi_{2} \approx \perp\right\}$. Suppose that $\Delta \sqcup\left\{\chi_{1} \vee \chi_{2} \approx\right.$ $\perp\} \models_{\mathbf{F}_{\mathrm{DMA}}} \varphi \approx \psi$. Then also $\Delta \cup\left\{\chi_{1} \approx \perp, \chi_{2} \approx\right.$ $\perp\} \models_{\mathbf{F}_{\mathrm{DMA}}} \varphi \approx \psi$. So by the induction hypothesis, $\Delta \cup$ $\left\{\chi_{1} \approx \perp, \chi_{2} \approx \perp\right\} \models_{\text {DMA }^{*}} \varphi \approx \psi$. But then since $\left\{\chi_{1} \vee\right.$ $\left.\chi_{2} \approx \perp\right\} \models \mathrm{DMA}^{*} \chi_{i} \approx \perp$ for $i=1,2$, we obtain $\Delta \sqcup$ $\left\{\chi_{1} \vee \chi_{2} \approx \perp\right\} \models_{\mathrm{DMA}^{*}} \varphi \approx \psi$ as required.

- $\Sigma=\Delta \sqcup\left\{\chi_{1} \vee \chi_{2} \approx \top\right\}$. Suppose that $\Delta \sqcup\left\{\chi_{1} \vee \chi_{2} \approx\right.$ $\top\} \models_{\mathbf{F}_{\mathrm{DMA}}} \varphi \approx \psi$. Then $\Delta \cup\left\{\chi_{i} \approx \top\right\} \models_{\mathbf{F}_{\mathrm{DMA}}} \varphi \approx \psi$ for $i=1,2$. So by the induction hypothesis, $\Delta \cup\left\{\chi_{i} \approx\right.$
T\} $\models_{\mathrm{DMA}^{*}} \varphi \approx \psi$ for $i=1,2$. But now, since (3) holds in every algebra in $\mathrm{DMA}^{*}$, we have $\Delta \sqcup\left\{\chi_{1} \vee \chi_{2}\right.$ $\approx \top\} \models \operatorname{DMA}^{*} \varphi \approx \psi$ as required.

- $\Sigma=\Delta \sqcup\{\neg \chi \approx \top\}$. Suppose that $\Delta \sqcup\{\neg \chi \approx \top\} \models$ $\mathbf{F}_{\mathrm{DMA}} \varphi \approx \psi$. Then $\Delta \cup\{\chi \approx \perp\} \models F_{\mathbf{F}_{\mathrm{DMA}}} \varphi \approx \psi$, so by the induction hypothesis, $\Delta \cup\{\chi \approx \perp\} \vDash \operatorname{DMA}^{*} \varphi \approx \psi$. But then also $\Delta \sqcup\{\neg \chi \approx \top\} \models \mathrm{DMA}^{*} \varphi \approx \psi$ as required.

- $\Sigma=\Delta \sqcup\{x \approx \top\}$. Suppose that $\Delta \sqcup\{x \approx \top\} \models_{\mathbf{F}_{\mathrm{DMA}}}$ $\varphi \approx \psi$. Let $\Delta^{\prime}$ and $\varphi^{\prime} \approx \psi^{\prime}$ be the result of substituting every occurrence of $\top$ for $x$ in $\Delta$ and $\varphi \approx \psi$, respectively. Then $\Delta^{\prime} \models_{\mathbf{F}_{\mathrm{DMA}}} \varphi^{\prime} \approx \psi^{\prime}$. Notice that $c\left(\Delta^{\prime}\right)=c(\Sigma)$ and $s\left(\Delta^{\prime}\right)<s(\Sigma)$. By Lemma 7, we can find identities $\Delta^{*}$ and $\varphi^{*} \approx \psi^{*}$ in normal form such that

1. $\Delta^{*} \models_{\mathbf{F}_{\mathrm{DMA}}} \varphi^{*} \approx \psi^{*}$

2. $c\left(\Delta^{*}\right) \leq c\left(\Delta^{\prime}\right)$ and $s\left(\Delta^{*}\right)=s\left(\Delta^{\prime}\right)$

3. $\Delta^{*} \models \mathrm{DMA}^{*} \varphi^{*} \approx \psi^{*}$ implies $\Delta \cup\{x \approx \top\} \models \mathrm{DMA}^{*}$ $\varphi \approx \psi$.

By the induction hypothesis, using 1. and 2., $\Delta^{*} \models \mathrm{DMA}^{*} \varphi^{*} \approx \psi^{*}$. But then also by $3 ., \Delta \cup\{x \approx$ T\} $\models \operatorname{DMA}^{*} \varphi \approx \psi$ as required.

- The cases $\Sigma=\Delta \sqcup\left\{\chi_{1} \wedge \chi_{2} \approx \perp\right\}, \Sigma=\Delta \sqcup\left\{\chi_{1} \wedge \chi_{2}\right.$ $\approx \top\}, \Sigma=\Delta \sqcup\{\neg \chi \approx \perp\}$, and $\Sigma=\Delta \sqcup\{x \approx \perp\}$ are treated symmetrically to the preceding cases.

We remark that this result leaves open two interesting questions: Can we find a similarly elegant basis of quasiequations for the admissible quasi-identities of DMA? And does $\{(1),(3)\}$ axiomatize the universal theory of $\mathbf{F}_{\text {DMA }}$ relative to DMA? I.e., is it the case that $\Sigma \Rightarrow \Delta$ holds in $\mathbf{F}_{\text {DMA }}$ iff $\Sigma \Rightarrow \Delta$ holds in all De Morgan algebras in which (1) and (3) both hold?

\section{Related work}

De Morgan lattices can be regarded as the algebraic counterpart of Belnap's four-valued logic (see Font 1997 for details). However, since there exists no faithful translation of equations into formulas of this logic, DML is not the equivalent algebraic semantics of this or of any algebraizable logic (Font 1997, Proposition 2.12). Indeed, Belnap's logic has no theorems so admissibility is trivial: every rule with at least one premise is admissible. Nevertheless, the algebras of many notable (substructural and many-valued) logics have De Morgan algebras or De Morgan lattices as reducts, in particular, the algebras of multiplicative additive linear logic, the relevant logics $\mathrm{R}$ and R-Mingle, and Łukasiewicz logics (see, e.g., Galatos et al. 2007). In this final section, we briefly survey the state of the art regarding questions of structural completeness and admissible rules for these and related classes of algebras. 
An involutive commutative residuated lattice (involutive $C R L$ for short) is an algebra $\mathbf{A}=\langle A, \wedge, \vee, \cdot, \rightarrow, \neg, \mathrm{t}\rangle$ with binary operations $\wedge, \vee, \cdot, \rightarrow$, a unary operation $\neg$, and a constant $\mathrm{t}$ such that $(1)\langle A, \wedge, \vee, \neg\rangle$ is a De Morgan lattice; (2) $\langle A, \cdot, \mathrm{t}\rangle$ is a commutative monoid; (3) $x \rightarrow y=\neg(x \cdot \neg y)$ for all $x, y \in A$. A bounded involutive $C R L$ is an algebra $\mathbf{A}=\langle A, \wedge, \vee, \cdot, \rightarrow, \neg, \mathrm{t}, \perp, \top\rangle$ such that $\langle A, \wedge, \vee, \cdot, \rightarrow, \neg, \mathrm{t}\rangle$ is an involutive CRL and $\langle A, \wedge, \vee, \neg, \perp, \top\rangle$ is a De Morgan algebra. We also define $x^{0}=\mathrm{t}$ and $x^{n+1}=x \cdot x^{n}$ for $n \in \mathbb{N}$.

It is easy to see (following a similar proof by Cintula and Metcalfe 2009) that the variety of involutive CRLs is not structurally complete. For $3 \leq n \in \mathbb{N}$, let

$L_{n}=\{0,1 /(n-1), \ldots,(n-2) /(n-1), 1\} \quad$ and

$\mathrm{七}_{\mathrm{n}}=\left\langle L_{n}, \min , \max ,{ }_{\mathrm{t}}, \rightarrow_{\mathrm{t}}, \neg_{\mathrm{七}}, 1\right\rangle$

where $x \cdot_{七} y=\max (x+y-1,0), x \rightarrow_{€} y=\min (1,1-x+y)$,

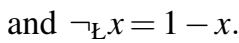

Lemma 8 Let $\mathcal{Q}$ be a quasivariety of involutive CRLs. If $\mathrm{七}_{n} \in \mathcal{Q}$ for some $3 \leq n \in \mathbb{N}$, then $\mathcal{Q}$ is not structurally complete.

Proof We simply note that the quasi-identity

$x^{n-1} \leq \neg x, \neg x \leq x \Rightarrow x \approx y$

does not hold in $\mathcal{Q}$ since it does not hold in $\mathfrak{七}_{\mathbf{n}}$ (just let $x=(n-2) /(n-1)$ and $y=1)$. On the other hand, note that if $\varphi^{n-1} \leq \neg \varphi$ and $\neg \varphi \leq \varphi$ hold in all members of $\mathcal{Q}$ for some formula $\varphi$, then $\varphi=\neg \varphi$ holds in the two-valued algebra $Ł_{2}$. But this is not possible, so the quasi-identity is admissible in $\mathcal{Q}$.

In particular, the class of (bounded) involutive CRLs (the algebras of multiplicative additive linear logic) is not structurally complete. Further general results for structural completeness and its failures for classes of algebras for substructural and many-valued logics may be found in the recent papers of Olson et al. (2008) and Cintula and Metcalfe (2009). However, more precise characterizations of admissibility for members of this family where structural completeness fails have so far been limited to some rather special classes of algebras.

Sugihara monoids, the algebras of the logic R-Mingle are involutive CRLs satisfying $x \approx x \cdot x$ and $x \wedge(y \vee z) \approx$ $(x \wedge y) \vee(x \wedge z)$. A (quite complicated) proof of structural completeness for the class of positive Sugihara monoids (the $\neg$-free subreducts of Sugihara monoids) was given in Olson and Raftery (2007). However, as is well known, the class of Sugihara monoids is not structurally complete as is shown by the admissible quasi-identity

$\mathrm{t} \leq(x \wedge \neg x) \vee y \Rightarrow \mathrm{t} \leq y$.

$M V$-algebras, the algebras of Łukasiewicz logic(s), are term-equivalent to involutive CRLs satisfying $(x \rightarrow y) \rightarrow y \approx x \vee y$. The $n$-valued Łukasiewicz logic corresponds to $\mathbb{Q}\left(€_{\mathbf{n}}\right)$, and the infinite-valued Łukasiewicz logic to the class of all MV-algebras (generated as a quasivariety by $\left\langle[0,1]\right.$, min, $\left.\left.\max ,{ }^{\cdot},, \rightarrow_{七}, \neg_{七}, 1\right\rangle\right)$. In Jeřábek (2010a) it was shown by Jeřábek (in a logical setting) that a basis for the admissible quasi-identities of $\mathbb{Q}\left(€_{\mathbf{n}}\right)$ is provided by $\neg(x \vee \neg x)^{n} \approx \mathrm{t} \Rightarrow x \approx y$.

Jeřábek has also given a more complicated basis for the admissible quasi-identities of the whole class of MV-algebras (Jeřábek 2010b). On the other hand, the class of implicational subreducts of MV-algebras is structurally complete. A proof may be found in Cintula and Metcalfe (2009), as can a proof that the class of $\{\rightarrow, \cdot\}$-subreducts is not structurally complete (however, no basis has yet been found). It is also shown in this paper that the varieties corresponding to Gödel logic, product logic, and the implicational fragment of Hájek's Basic logic (but not the full logic) are structurally complete.

Acknowledgments We are grateful for the helpful comments of both the anonymous referees and Leonardo Cabrer. The authors acknowledge support from Swiss National Science Foundation Grant 20002_129507.

\section{References}

Bergman C (1991) Structural completeness in algebra and logic. In: Andréka H, Monk J, Nemeti I (eds) Algebraic logic (proc. conf., Budapest, 8-14 August 1988), Colloquia Mathematica Societatis János Bolyai, vol 54. North-Holland, Amsterdam, pp 59-73

Burris S, Sankappanavar HP (1981) A course in universal algebra. Graduate texts in mathematics, vol 78. Springer, New York

Cintula P, Metcalfe G (2009) Structural completeness in fuzzy logics. Notre Dame J Formal Log 50(2):153-183

Cintula P, Metcalfe G (2010) Admissible rules in the implicationnegation fragment of intuitionistic logic. Ann Pure Appl Log 162(10):162-171

Font JM (1997) Belnap's four-valued logic and De Morgan lattices. Log J IGPL 5(3):1-29

Gaitán H, Perea MH (2004) A non-finitely based quasi-variety of De Morgan algebras. Stud Log 78(1-2):237-248

Galatos N, Jipsen P, Kowalski T, Ono H (2007) Residuated lattices: an algebraic glimpse at substructural logics. Elsevier, Amsterdam

Gehrke M, Walker CL, Walker EA (2003) Normal forms and truth tables for fuzzy logics. Fuzzy Sets Syst 138:25-51

Ghilardi S (1999) Unification in intuitionistic logic. J Symb Log 64(2):859-880

Ghilardi S (2000) Best solving modal equations. Ann Pure Appl Log 102(3): 184-198

Iemhoff R (2001) On the admissible rules of intuitionistic propositional logic. J Symb Log 66(1):281-294

Jeřábek E (2005) Admissible rules of modal logics. J Log Comput $15: 411-431$

Jeřábek E (2010a) Admissible rules of Łukasiewicz logic. J Log Comput 20(2):425-447

Jeřábek E (2010b) Bases of admissible rules of Łukasiewicz logic. J Log Comput 20(6):1149-1163 
Kalman JA (1958) Lattices with involution. Trans Am Math Soc 87:485-491

Moisil G (1935) Recherches sur l'algébre de la logique. Ann Sci Univ Jassy 22(3):1-117

Olson JS, Raftery JG (2007) Positive Sugihara monoids. Algebra Univers 57:75-99

Olson JS, Raftery JG, Alten CJV (2008) Structural completeness in substructural logics. Log J IGPL 16(5):453-495
Pynko AP (1999) Implicational classes of De Morgan lattices. Discrete Math 205(1-3):171-181

Rybakov VV (1997) Admissibility of logical inference rules. Studies in logic and the foundations of mathematics, vol 136. Elsevier, Amsterdam 